\title{
Glucosamine Sulfate/Chondroitin Sulfate Tablet
}

National Cancer Institute

\section{Source}

National Cancer Institute. Glucosamine Sulfate/Chondroitin Sulfate Tablet. NCI

Thesaurus. Code C77881.

An oral tablet formulation of a dietary supplement containing the sulfate salts of the amino sugar glucosamine and the glucosaminoglycan chondroitin with potential analgesic, anti-inflammatory, and anti-arthritic activities. Both glucosamine and chondroitin are naturally occurring substances that play a key role in cartilage formation and repair. Glucosamine is an essential substrate for glycosaminoglycans and hyaluronic acid, needed for formation of the joint proteoglycan structural matrix, and may prevent chondrocytic catabolic activity and inhibit production of inflammatory mediators. Chondroitin is the major glycosaminoglycan in cartilage, responsible for the elasticity of cartilage; it may reduce the IL-1 beta-induced nuclear factor-kappaB (NF-kB) translocation in chondrocytes, inhibiting NF-kB-mediated inflammatory processes. 